\title{
On transfer homomorphisms of Krull monoids
}

\author{
Alfred Geroldinger ${ }^{1}$ (D) $\cdot$ Florian Kainrath $^{1}$
}

Received: 1 May 2021 / Accepted: 17 June 2021 / Published online: 28 June 2021

(c) The Author(s) 2021, corrected publication 2021

\begin{abstract}
Every Krull monoid has a transfer homomorphism onto a monoid of zero-sum sequences over a subset of its class group. This transfer homomorphism is a crucial tool for studying the arithmetic of Krull monoids. In the present paper, we strengthen and refine this tool for Krull monoids with finitely generated class group.
\end{abstract}

Keywords Transfer homomorphisms · Krull monoids · Transfer Krull monoids · Class groups

Mathematics Subject Classification 20M12 $\cdot 20 \mathrm{M} 13 \cdot 13 \mathrm{~A} 15 \cdot 13 \mathrm{~F} 05 \cdot 16 \mathrm{D} 70$

\section{Introduction}

Transfer homomorphisms are a central tool in factorization theory. Since they are essentially surjective and allow to lift factorizations, they make it possible to study the arithmetic of monoids and domains as follows. Given an object $H$ of interest, find a simpler object $B$, and a transfer homomorphism $\theta: H \rightarrow B$; study the arithmetic of the simpler object $B$ and then pull back arithmetical results from $B$ to the original object of interest $H$. Oftentimes, the object $B$ is constructed with divisor theoretic or ideal theoretic methods and it has the same algebraic structure as $H$ but a simpler combinatorial structure. This holds true, in particular, for Krull monoids, weakly Krull monoids, and C-monoids, and for these classes of monoids the described strategy has turned out to be highly efficient. We refer to [20] for background on transfer homomorphisms.

In the present paper we focus on transfer homomorphisms of Krull monoids. Since a domain $D$ is a Krull domain if and only if its multiplicative monoid $D \backslash\{0\}$ of nonzero

The original online version of this article was revised to add funding note.

$\bowtie \quad$ Alfred Geroldinger

alfred.geroldinger@uni-graz.at

Florian Kainrath

florian.kainrath@uni-graz.at

https://imsc.uni-graz.at/geroldinger

1 Institute of Mathematics and Scientific Computing, University of Graz, NAWI Graz, Heinrichstraße 36, 8010 Graz, Austria 
elements is a Krull monoid, we also have the domain case in mind when speaking of Krull monoids. Let $H$ be a Krull monoid with divisor theory $\varphi: H \rightarrow \mathcal{F}(P)$, class group $G$, and let $G_{P} \subset G$ denote the set of classes containing prime divisors. Then the monoid $\mathcal{B}\left(G_{P}\right)$ of zero-sum sequences over $G_{P}$ is a Krull monoid again, and there is a canonical transfer homomorphism $\theta: H \rightarrow \mathcal{B}\left(G_{P}\right)$. It is an easy observation to see that the inclusion $\mathcal{B}\left(G_{P}\right) \hookrightarrow \mathcal{F}\left(G_{P}\right)$, where $\mathcal{F}\left(G_{P}\right)$ is the free abelian monoid with basis $G_{P}$, is a cofinal divisor homomorphism but in general not a divisor theory. This means that $\mathcal{B}\left(G_{P}\right)$ is not optimal, neither from the algebraic point of view nor for arithmetical investigations.

It turned out, first for special subsets of finite abelian groups, that it is possible to construct a new subset $G_{P}^{\prime}$ and a divisor homomorphism $\theta^{\prime}: H \rightarrow \mathcal{B}\left(G_{P}^{\prime}\right)$ such that $\mathcal{B}\left(G_{P}^{\prime}\right) \hookrightarrow$ $\mathcal{F}\left(G_{P}^{\prime}\right)$ is a divisor theory. Such refinement constructions are used in a variety of arithmetical investigations of $H$ (for a sample, see [33, Theorem 3.17], [34, Theorem 3.1], [20, Chap. 6.7], [32, Lemma 4.5]). In [35], Schmid proved that for every Krull monoid $H$ with torsion class group $G$ there is a subset $G_{0}^{*} \subset G$, and a transfer homomorphism $\theta^{*}: H \rightarrow \mathcal{B}\left(G_{0}^{*}\right)$ such that $\mathcal{B}\left(G_{0}^{*}\right) \hookrightarrow \mathcal{F}\left(G_{0}^{*}\right)$ is a divisor theory (Proposition 3.3). This was done by transfinite induction over a combinatorial construction. In this paper, we show the same result for Krull monoids with finitely generated class group (Theorem 3.4). In contrast to the result by Schmid, we use ideal and divisor theoretic methods.

In Sect. 2, we gather the necessary background on Krull monoids. Our main result (Theorem 3.4) is proved in Sect. 3. In the last section, we apply our results to transfer Krull monoids (Theorem 4.3) and to weakly Krull monoids (Theorem 4.6). Finally, we demonstrate the usefulness of our construction in an example of a Krull monoid with infinite class group (Example 4.10).

\section{Background on Krull monoids}

Arithmetic of monoids. By a monoid, we mean a semigroup with identity element. If not stated otherwise, we use multiplicative notation. Let $H$ be a monoid with identity $1_{H} \in H$, and let $H^{\times}$denote the group of invertible elements. We say that $H$ is

- unit-cancellative if $a, u \in H$ and $a=a u$ or $a=u a$ implies $u \in H^{\times}$;

- cancellative if $a, b, c \in H$ and $a b=a c$ or $b a=c a$ implies $b=c$.

A non-unit $a \in H$ is said to be an atom (or irreducible) if $b, c \in H$ and $a=b c$ implies that $b \in H^{\times}$or $c \in H^{\times}$. We denote by $\mathcal{A}(H)$ the set of atoms of $H$ and we say that $H$ is atomic if every non-unit is a finite product of atoms. If $a=u_{1} \cdot \ldots \cdot u_{k} \in H$ is a product of $k$ atoms, then $k$ is called a factorization length of $a$ and the set $\mathrm{L}_{H}(a)=\mathrm{L}(a) \subset \mathbb{N}$ is the set of lengths of $a$. It is convenient to set $\mathrm{L}(u)=\{0\}$ for all $u \in H^{\times}$. Then

$$
\mathcal{L}(H)=\{\mathrm{L}(a) \mid a \in H\}
$$

denotes the system of sets of lengths of $H$ and

$$
\Delta(H)=\bigcup_{L \in \mathcal{L}(H)} \Delta(L) \subset \mathbb{N}
$$

denotes the set of distances of $H$, where $\Delta(L)$ is the set of successive distances of $L$. For a set $P$, let $\mathcal{F}(P)$ denote the free abelian monoid with basis $P$. Then every $a \in \mathcal{F}(P)$ has a unique representation in the form

$$
a=\prod_{p \in P} p^{\mathrm{v}_{p}(a)},
$$


where $\mathrm{v}_{p}: \mathcal{F}(P) \rightarrow \mathbb{N}_{0}$ is the $p$-adic valuation of $a$. We call $|a|=\sum_{p \in P} \mathrm{v}_{p}(a) \in \mathbb{N}_{0}$ the length of $a$ and $\operatorname{supp}(a)=\left\{p \in P \mid \mathrm{v}_{p}(a)>0\right\} \subset P$ the support of $a$.

Now suppose that $H$ is a commutative unit-cancellative monoid. We say that $H$ is reduced if $H^{\times}=\left\{1_{H}\right\}$, and we denote by $H_{\text {red }}=\left\{a H^{\times} \mid a \in H\right\}$ the associated reduced monoid of $H$. The free abelian monoid $\mathrm{Z}(H)=\mathcal{F}\left(\mathcal{A}\left(H_{\text {red }}\right)\right)$ is the factorization monoid of $H$, and we denote by $\pi: Z(H) \rightarrow H_{\text {red }}$ the canonical epimorphism. For an element $a \in H$,

$$
\mathrm{Z}(a)=\pi^{-1}\left(a H^{\times}\right) \subset \mathrm{Z}(H) \text { is the set of factorizations of } a .
$$

To define a distance function on $\mathrm{Z}(H)$, let

$$
z=u_{1} \cdot \ldots \cdot u_{k} v_{1} \cdot \ldots \cdot v_{\ell} \text { and } z^{\prime}=u_{1} \cdot \ldots \cdot u_{k} w_{1} \cdot \ldots \cdot w_{m}
$$

be two factorizations, where $k, \ell, m \in \mathbb{N}_{0}$, all $u_{r}, v_{s}, w_{t} \in \mathcal{A}\left(H_{\text {red }}\right)$ such that $v_{s} \neq w_{t}$ for all $s \in[1, \ell]$ and $t \in[1, m]$. Then $\mathrm{d}\left(z, z^{\prime}\right)=\max \{\ell, m\} \in \mathbb{N}_{0}$ is the distance between $z$ and $z^{\prime}$. Let $a \in H$ and $M \in \mathbb{N}_{0}$. A sequence $z_{0}, \ldots, z_{k} \in \mathrm{Z}(a)$ is called an $M$-chain of factorizations if $\mathrm{d}\left(z_{i-1}, z_{i}\right) \leq M$ for all $i \in[1, k]$. The catenary degree $\mathrm{c}(a)$ is the smallest $M \in \mathbb{N}_{0} \cup\{\infty\}$ such that any two factorizations $z, z^{\prime} \in \mathrm{Z}(a)$ can be concatenated by an $M$-chain. Then

$$
\mathrm{c}(H)=\sup \{\mathrm{c}(a) \mid a \in H\} \in \mathbb{N}_{0} \cup\{\infty\}
$$

is the catenary degree of $H$. For $u \in \mathcal{A}\left(H_{\text {red }}\right)$, the local tame degree $\mathrm{t}(H, u)$ is the smallest $M \in \mathbb{N}_{0} \cup\{\infty\}$ having the following property: if $a \in H$ with $\mathrm{Z}(a) \cap u \mathrm{Z}(H) \neq \varnothing$ and $z \in \mathrm{Z}(a)$, then there is $z^{\prime} \in \mathrm{Z}(a) \cap u \mathrm{Z}(H)$ such that $\mathrm{d}\left(z, z^{\prime}\right) \leq M$. The monoid $H$ is called

- locally tame if $\mathrm{t}(H, u)<\infty$ for all $u \in \mathcal{A}\left(H_{\text {red }}\right)$ and

- (globally) tame if $\mathrm{t}(H)=\sup \left\{\mathrm{t}(H, u) \mid u \in \mathcal{A}\left(H_{\text {red }}\right)\right\}<\infty$.

Krull monoids. We gather the basics of Krull monoids (detailed presentations can be found in [20,29]). Let $H$ and $D$ be commutative and cancellative monoids and let $\varphi: H \rightarrow D$ be a monoid homomorphism. Then $\varphi$ can be uniquely lifted to a group homomorphism $\mathrm{q}(\varphi): \mathrm{q}(H) \rightarrow \mathrm{q}(D)$, where $\mathrm{q}(H)$ and $\mathrm{q}(D)$ denote the quotient groups of $H$ and $D$. Moreover, $\varphi$ is called

- a divisor homomorphism if $\varphi(a) \mid \varphi(b)$ implies that $a \mid b$ for all $a, b \in H$;

- cofinal if for every $a \in D$ there exists some $u \in H$ such that $a \mid \varphi(u)$;

- a divisor theory (for $H$ ) if $D=\mathcal{F}(P)$ for some set $P, \varphi$ is a divisor homomorphism, and for every $p \in P$ (equivalently for every $a \in \mathcal{F}(P)$ ), there exists a finite subset $\emptyset \neq X \subset H$ satisfying $p=\operatorname{gcd}(\varphi(X))$.

We call $\mathcal{C}(\varphi)=\mathrm{q}(D) / \mathrm{q}(\varphi(H))$ the class group of $\varphi$ and use additive notation for this group. For $a \in \mathrm{q}(D)$, we denote by $[a]=[a]_{\varphi}=a \mathrm{q}(\varphi(H)) \in \mathrm{q}(D) / \mathrm{q}(\varphi(H))$ the class containing $a$. The homomorphism $\varphi$ is cofinal if and only if $\mathcal{C}(\varphi)=\{[a] \mid a \in D\}$, and if $\varphi$ is a divisor homomorphism, then $\varphi(H)=\{a \in D \mid[a]=[1]\}$. If $\varphi: H \rightarrow \mathcal{F}(P)$ is a cofinal divisor homomorphism, then

$$
G_{P}=\{[p]=p q(\varphi(H)) \mid p \in P\} \subset \mathcal{C}(\varphi)
$$

is called the set of classes containing prime divisors, and we have $\left[G_{P}\right]=\mathcal{C}(\varphi)$.

Suppose that $H \subset D$ and that $\varphi=(H \hookrightarrow D)$. Then, $\mathcal{C}(\varphi)=\mathrm{q}(D) / \mathrm{q}(H)$, and for $a \in D$ we set $[a]_{\varphi}=[a]_{D / H}=a \mathrm{q}(H)$. Then

$$
D / H=\left\{[a]_{D / H} \mid a \in D\right\} \subset \mathcal{C}(\varphi)
$$


is a submonoid with quotient group $\mathrm{q}(D / H)=\mathcal{C}(\varphi)$. It is easy to check that $D / H$ is a group if and only if $H \hookrightarrow D$ is cofinal. In particular, if $D / H$ is finite or if $\mathrm{q}(D) / \mathrm{q}(H)$ is a torsion group, then $D / H=\mathrm{q}(D) / \mathrm{q}(H)$.

For subsets $A, B \subset \mathrm{q}(H)$, we denote by $(A: B)=\{x \in \mathrm{q}(H) \mid x B \subset A\}$, by $A^{-1}=$ $(H: A)$, and by $A_{v}=\left(A^{-1}\right)^{-1}$. A subset $\mathfrak{a} \subset H$ is an $s$-ideal of $H$ if $\mathfrak{a} H=\mathfrak{a}$. A subset $X \subset \mathrm{q}(H)$ is a fractional $v$-ideal (or a fractional divisorial ideal) if there is a $c \in H$ such that $c X \subset H$ and $X_{v}=X$. We denote by $\mathcal{F}_{v}(H)$ the set of all fractional $v$-ideals and by $\mathcal{I}_{v}(H)$ the set of all $v$-ideals of $H$. For $A, B \in \mathcal{F}_{v}(H)$, we define $A{ }_{v} B=(A B)_{v}$, and we call $A \cdot{ }_{v} B$ the $v$-product of $A$ and $B$. Furthermore, $\mathcal{I}_{v}^{*}(H)$ is the monoid of $v$-invertible $v$-ideals (with $v$-multiplication) and $\mathcal{F}_{v}(H)^{\times}=\mathrm{q}\left(\mathcal{I}_{v}^{*}(H)\right)$ is its quotient group of fractional invertible $v$-ideals. The monoid $H$ is called $v$-noetherian if it satisfies the ascending chain condition on $v$-ideals. If $H$ is $v$-noetherian, then all sets of lengths are finite. We denote by $\mathfrak{X}(H)$ the set of all minimal non-empty prime $s$-ideals of $H$.

The map $\partial: H \rightarrow \mathcal{I}_{v}^{*}(H)$, defined by $\partial(a)=a H$ for each $a \in H$, is a cofinal divisor homomorphism. Thus, if $\mathcal{H}=\{a H \mid a \in H\}$ is the monoid of principal ideals of $H$, then we have a cofinal divisor homomorphism

$$
\mathcal{H} \hookrightarrow \mathcal{I}_{v}^{*}(H) \text { and } \mathcal{C}_{v}(H)=\mathcal{I}_{v}^{*}(H) / \mathcal{H}=\mathcal{F}_{v}(H)^{\times} / \mathbf{q}(\mathcal{H})
$$

is the $v$-class group of $H$. The monoid $H$ is called a Krull monoid if it satisfies one of the following equivalent conditions ( [20, Theorem 2.4.8]):

(a) $H$ is $v$-noetherian and completely integrally closed.

(b) $H$ has a divisor theory.

(c) There is a free abelian monoid $F$ such that $H_{\text {red }} \hookrightarrow F$ is a divisor homomorphism.

Let $H$ be a Krull monoid. Then a divisor theory $\varphi: H \rightarrow \mathcal{F}(P)$ is unique up to unique isomorphism. In particular, the class group $\mathcal{C}(\varphi)$ defined via a divisor theory $\varphi$ of $H$ and the subset of classes containing prime divisors depend only on $H$. Thus it is called the class group of $H$ and is denoted by $\mathcal{C}(H)$. If $H$ is a Krull monoid, then $\mathcal{I}_{v}^{*}(H)$ is a free abelian monoid with basis $\mathfrak{X}(H)=v$-spec $(H) \backslash\{\emptyset\}$, the map $\partial: H \rightarrow \mathcal{I}_{v}^{*}(H)$ is a divisor theory, and $\mathcal{C}(H)$ is isomorphic to the $v$-class group $\mathcal{C}_{v}(H)$ of $H$.

Main examples of Krull monoids stem from ring and module theory. We mention two examples and refer to [20,26] for more. To begin with, a commutative integral domain is Krull if and only its monoid of nonzero elements is a Krull monoid. Therefore, Condition (a) shows that every integrally closed noetherian domain is a Krull domain. To give an example from module theory, let $R$ be a ring and $\mathcal{C}$ be a small class of left $R$-modules that is closed under finite direct sums, direct summands, and isomorphisms. Then the set $\mathcal{V}(\mathcal{C})$ of isomorphism classes of modules from $\mathcal{C}$ is a commutative monoid, with operation induced by the direct sum. If the modules in $\mathcal{C}$ are not isomorphic to proper direct summands, then $\mathcal{V}(\mathcal{C})$ is unit-cancellative. If the endomorphism $\operatorname{rings}_{\operatorname{End}_{R}}(M)$ are semilocal for all modules $M$ from $\mathcal{C}$, then $\mathcal{V}(\mathcal{C})$ is a Krull monoid ( [10, Theorem 3.4], [13]).

Monoids of zero-sum sequences. Let $G$ be an additive abelian group and let $G_{0} \subset G$ be a subset. Then $\left[G_{0}\right] \subset G$ denotes the submonoid and $\left\langle G_{0}\right\rangle \subset G$ denotes the subgroup generated by $G_{0}$. Let $\mathrm{r}(G)$ be the torsion-free rank of $G$. In additive combinatorics, the elements of the free abelian monoid $\mathcal{F}\left(G_{0}\right)$ are called sequences over $G_{0}$ (they can be understood as finite unordered sequences of terms from $G_{0}$, with repetition allowed). For $S=g_{1} \cdot \ldots \cdot g_{\ell} \in \mathcal{F}\left(G_{0}\right)$, let $\sigma(S)=g_{1}+\cdots+g_{\ell} \in G$ denote the sum of $S$. Then the set

$$
\mathcal{B}\left(G_{0}\right)=\left\{S \in \mathcal{F}\left(G_{0}\right) \mid \sigma(S)=0\right\} \subset \mathcal{F}\left(G_{0}\right)
$$


is a submonoid of $\mathcal{F}\left(G_{0}\right)$, called the monoid of zero-sum sequences over $G_{0}$. We say that $G_{0}$ is condensed if for every $g \in G_{0}$ there is $B \in \mathcal{B}\left(G_{0}\right)$ with $\mathrm{v}_{g}(B)>0$. Thus, $G_{0}$ is condensed if and only if the inclusion $\mathcal{B}\left(G_{0}\right) \hookrightarrow \mathcal{F}\left(G_{0}\right)$ is cofinal. Since the inclusion $\mathcal{B}\left(G_{0}\right) \hookrightarrow \mathcal{F}\left(G_{0}\right)$ is a divisor homomorphism, $\mathcal{B}\left(G_{0}\right)$ is a Krull monoid by Condition (c) (given in the definition of Krull monoids). Moreover, by [20, Proposition 2.5.6], we have that

$\mathcal{B}\left(G_{0}\right) \hookrightarrow \mathcal{F}\left(G_{0}\right)$ is a divisor theory if and only if $\left\langle G_{0}\right\rangle=\left[G_{0} \backslash\{g\}\right]$ for every $\left.g \in G 62.2\right)$

If $G_{0}$ is condensed, then $\sigma: \mathcal{F}\left(G_{0}\right) \longrightarrow G$ induces by [20, Proposition 2.5.6.2] an isomorphism

$$
\mathcal{C}\left(\mathcal{B}\left(G_{0}\right) \hookrightarrow \mathcal{F}\left(G_{0}\right)\right) \longrightarrow\left[G_{0}\right]
$$

As usual, we set $\mathcal{L}\left(G_{0}\right):=\mathcal{L}\left(\mathcal{B}\left(G_{0}\right)\right), \mathrm{c}\left(G_{0}\right):=\mathrm{c}\left(\mathcal{B}\left(G_{0}\right)\right), \Delta\left(G_{0}\right):=\Delta\left(\mathcal{B}\left(G_{0}\right)\right)$, and so on.

\section{Transfer homomorphisms of Krull monoids}

We start with the definition of transfer homomorphisms in a not necessarily commutative setting (see [6, Definition 2.1]). We need the concept in this general setting in Section 4.

Definition 3.1 A monoid homomorphism $\theta: H \rightarrow B$ of atomic unit-cancellative monoids $H$ and $B$ is called a transfer homomorphism if it satisfies the following two properties:

(T 1) $B=B^{\times} \theta(H) B^{\times}$and $\theta^{-1}\left(B^{\times}\right)=H^{\times}$.

(T 2) If $u \in H, b, c \in B$ and $\theta(u)=b c$, then there exist $v, w \in H$ and $\varepsilon \in B^{\times}$such that $u=v w, \theta(v)=b \varepsilon^{-1}$, and $\theta(w)=\varepsilon c$.

It is easy to check that the composition of transfer homomorphisms is a transfer homomorphism again. Moreover, transfer homomorphisms allow to pull back arithmetical properties from $B$ to $H$. In particular, if $\theta: H \rightarrow B$ is a transfer homomorphism, then

$$
\mathrm{L}_{H}(a)=\mathrm{L}_{B}(\theta(a)) \text { for every } a \in H \text { and } \mathcal{L}(H)=\mathcal{L}(B) .
$$

Details can be found in [6, Sect. 2] and in [20, Chap. 3.2]. The next lemma describes the canonical transfer homomorphism from a Krull monoid to a monoid of zero-sum sequences ( [20, Chap. 3.4]).

Lemma 3.2 Let $H$ be a Krull monoid, $\varphi: H \rightarrow F=\mathcal{F}(P)$ be a cofinal divisor homomorphism, $G=\mathcal{C}(\varphi)$ its class group, and $G_{P} \subset G$ the set of classes containing prime divisors. Let $\widetilde{\boldsymbol{\beta}}: F \rightarrow \mathcal{F}\left(G_{P}\right)$ denote the unique homomorphism defined by $\widetilde{\boldsymbol{\beta}}(p)=[p]$ for all $p \in P$.

1. The homomorphism $\boldsymbol{\beta}_{H}=\boldsymbol{\beta}=\widetilde{\boldsymbol{\beta}} \circ \varphi: H \rightarrow \mathcal{B}\left(G_{P}\right)$ is a transfer homomorphism.

2. The inclusion $\mathcal{B}\left(G_{P}\right) \hookrightarrow \mathcal{F}\left(G_{P}\right)$ is a cofinal divisor homomorphism.

The homomorphism $\boldsymbol{\beta}$ in Lemma 3.2 is called the block homomorphism, and $\mathcal{B}\left(G_{P}\right)$ is called the block monoid associated to $\varphi$. If $\varphi$ is a divisor theory, then $\mathcal{B}\left(G_{P}\right)$ is called the block monoid associated to $H$. Block homomorphisms enable us to study the arithmetic of Krull monoids with methods from additive combinatorics (see [23, Chap. 1] and [15,36] for surveys on this interaction).

If the inclusion $\mathcal{B}\left(G_{P}\right) \hookrightarrow \mathcal{F}\left(G_{P}\right)$ is a divisor theory, then $\varphi: H \rightarrow \mathcal{F}(P)$ is a divisor theory. However, in a variety of settings, the fact that the monoid under consideration is Krull, 
is proved by establishing a (cofinal) divisor homomorphism to a free abelian monoid, which in general need not be a divisor theory. Moreover, oftentimes there is no information on the class group of the Krull monoid but just on the class group of the divisor homomorphism (see [10, Theorem 3.4] for an example from module theory and [37, Proposition 3.17] for an example from ring theory).

The question, which pairs $\left(G, G_{P}\right)$ can actually occur, is answered by the following realization result ( [20, Theorems 2.5.4 and 3.7.8]). Let $G$ be an abelian group and $G_{0} \subset G$ be a subset. Then the following statements are equivalent.

(a) There is a Dedekind domain $D$ with class group $G$ such that $G_{0}$ is the set of classes containing non-zero prime ideals.

(b) There is a Krull monoid $H$ with class group $G$ such that $G_{0}$ is the set of classes containing prime divisors.

(c) $G=\left[G_{0}\right]$.

Comparing this result with (2.2) we see that, in general, the inclusion $\mathcal{B}\left(G_{P}\right) \hookrightarrow \mathcal{F}\left(G_{P}\right)$, given in Lemma 3.2, is not a divisor theory. By Condition (a), this need not even be the case when the homomorphism $\varphi$ (in Lemma 3.2) is a divisor theory. If $G_{P}$ consists of torsion elements, then there is the following result.

Proposition 3.3 Let $H$ be a Krull monoid with torsion class group $G$. Then there is a subset $G_{0}^{*} \subset G$ such that $\mathcal{B}\left(G_{0}^{*}\right) \hookrightarrow \mathcal{F}\left(G_{0}^{*}\right)$ is a divisor theory and a transfer homomorphism $\theta^{*}: H \rightarrow \mathcal{B}\left(G_{0}^{*}\right)$.

Proof Let $G_{P} \subset G$ denote the set of classes containing prime divisors. By [35, Corollary 4.13], there is a subset $G_{0}^{*} \subset G$ such that $g \in\left\langle G_{0}^{*} \backslash\{g\}\right\rangle$ for all $g \in G_{0}^{*}$ and a transfer homomorphism $\theta: \mathcal{B}\left(G_{P}\right) \rightarrow \mathcal{B}\left(G_{0}^{*}\right)$ (if $G_{0}$ is finite, this follows from a simple combinatorial argument given in [20, Theorem 6.7.11]). Thus Eq. (2.2) implies that $\mathcal{B}\left(G_{0}^{*}\right) \hookrightarrow \mathcal{F}\left(G_{0}^{*}\right)$ is a divisor theory. If $\boldsymbol{\beta}: H \rightarrow \mathcal{B}\left(G_{P}\right)$ is the transfer homomorphism given in Lemma 3.2, then $\theta^{*}=\theta \circ \boldsymbol{\beta}: H \rightarrow \mathcal{B}\left(G_{0}^{*}\right)$ is a transfer homomorphism too.

The goal of this section is to obtain a result as given in Proposition 3.3 for Krull monoids with finitely generated class group. Starting from cases where the torsion-free rank of the class group equals one $[7,8,19,38]$, the arithmetic of Krull monoids with finitely generated class group has seen strong interest (e.g., [17,27]), partly motivated by module theory $[4,9,11-$ 13]. Moreover, the refinement of the block homomorphism, as given in Proposition 3.3 and Theorem 3.4, is also used for studying the arithmetic of Krull monoids which have prime divisors in all classes (see Remark 3.10). We formulate our main result.

Theorem 3.4 Let $H$ be a Krull monoid with finitely generated class group. Then there exist a finitely generated abelian group $G^{*}$, a subset $G_{0}^{*} \subset G^{*}$ such that $\mathcal{B}\left(G_{0}^{*}\right) \hookrightarrow \mathcal{F}\left(G_{0}^{*}\right)$ is a divisor theory, and a transfer homomorphism $\theta^{*}: H \rightarrow \mathcal{B}\left(G_{0}^{*}\right)$, which is a composition of block homomorphisms. In particular, we have

1. $\mathcal{L}(H)=\mathcal{L}\left(G_{0}^{*}\right)$.

2. $c\left(G_{0}^{*}\right) \leq c(H) \leq \max \left\{c\left(G_{0}^{*}\right), 2\right\}$.

3. $H$ is locally tame if and only if $\mathcal{B}\left(G_{0}^{*}\right)$ is locally tame.

To prove Theorem 3.4, we need some preparations. First we construct, for a given reduced Krull monoid $H$ and a divisor homomorphism to a free abelian monoid, a divisor theory for $H$. Such a construction has already been given in [20, Theorem 2.4.7], but this construction is not explicit enough for our purpose. So, let $H$ be a reduced Krull monoid and $F=\mathcal{F}(P)$ be a free abelian monoid such that the inclusion $H \hookrightarrow F$ is a divisor homomorphism. We say that an element $x \in F$ is irrelevant if $(x F \cap H)_{v}=H$. 
Lemma 3.5 1. The product of irrelevant elements is irrelevant, too.

2. Let $p \in P$ be not irrelevant. Then $p F \cap H \in v-\operatorname{spec}(H)$.

3. Let $q \in \mathfrak{X}(H)$. Then there exists some $p \in P$, such that $q=p F \cap H$.

Proof 1. If $x, y \in F$ are irrelevant, then

$$
H=H \cdot{ }_{v} H=(x F \cap H)_{v} \cdot v(y F \cap H)_{v}=((x F \cap H)(y F \cap H))_{v} \subset(x y F \cap H)_{v} \subset H .
$$

2. If $p F \cap H=\emptyset$, we are done. Hence we may assume $p F \cap H \neq \emptyset$. Then $p F \cap H$ is a non-empty prime $s$-ideal of $H$, and hence contains some minimal, non-empty, prime $s$-ideal $q$. Then $q \in \mathfrak{X}(H)$. Now we have

$$
q \subset p F \cap H \subset(p F \cap H)_{v} \neq H .
$$

Since $H$ is Krull, $q$ is a maximal $v$-ideal, which implies $q=p F \cap H$.

3. Let $x=\operatorname{gcd}(q) \in F$. By [20, Proposition 2.4.2.6] we have $q=x F \cap H$. Therefore $x$ is not irrelevant. Now let $p_{1}, \ldots, p_{n} \in P$ be such that $x=p_{1} \ldots p_{n}$. By Lemma 3.5.1 we may assume that $p_{1}$ is not irrelevant. Then have

$$
q=x F \cap H \subset p_{1} F \cap H .
$$

By 1 . we know, that $p_{1} F \cap H$ is a $v$-ideal, not equal to $H$. Since $q$ is $v$-maximal, we obtain $q=p_{1} F \cap H$.

By Lemma 3.5.3, we may choose a map $f: \mathfrak{X}(H) \rightarrow P$ such that $q=f(q) F \cap H$ for each $q \in \mathfrak{X}(H)$ (in particular $f$ is injective). Now let $q \in \mathfrak{X}(H)$ and let

$$
\mathrm{v}_{q}: \mathrm{q}(H) \rightarrow \mathbb{Z}, \quad \mathrm{v}_{f(q)}: \mathrm{q}(F) \rightarrow \mathbb{Z}
$$

be the corresponding valuations. From $q=f(q) F \cap H$ we obtain $H_{q}^{\times}=H_{q} \cap F_{f(q) F}^{\times}$. Hence, if $\pi$ is a prime of $H_{q}$ (which is a discrete valuation monoid), we have $\pi=\varepsilon f(q)^{e(q)}$ for some $\varepsilon \in F_{f(q) F}^{\times}$and some $e(q) \in \mathbb{N}$. This implies

$$
\mathrm{v}_{f(q)}(h)=e(q) \mathrm{v}_{q}(h)
$$

for all $h \in \mathrm{q}(H)$. Therefore, we obtain that

$$
\mathrm{v}_{f(q)}(H)=e(q) \mathrm{v}_{q}(H)=e(q) \mathbb{N}_{0}
$$

and

$$
e(q)=\min \mathbf{v}_{f(q)}(H) \backslash\{0\}=\operatorname{gcd}\left(\mathbf{v}_{f(q)}(H)\right) .
$$

We define

$$
\begin{gathered}
P_{0}=f(\mathfrak{X}(H)) \subset P, \quad F_{0}=\left[\left\{f(q)^{e(q)} \mid q \in \mathfrak{X}(H)\right\} \cup P \backslash P_{0}\right], \quad \text { and } \\
F_{1}=\left[\left\{f(q)^{e(q)} \mid q \in \mathfrak{X}(H)\right\}\right] .
\end{gathered}
$$

By (3.2), we have $H \subset F_{0}$. Since $H \hookrightarrow F$ is a divisor homomorphism, the same is true for $H \hookrightarrow F_{0}$. We define $d: F_{0} \rightarrow F_{1}$ by

$$
d_{\mid F_{1}}=\operatorname{id}_{F_{1}}, \quad d(p)=1, \text { for all } p \in P \backslash P_{0}
$$

and set $\partial=d_{\mid H}: H \rightarrow F_{1}$.

Note that $F_{1}$ is free with basis $\left\{f(q)^{e(q)} \mid q \in \mathfrak{X}(H)\right\}$. Hence $f$ induces an isomorphism $f_{*}: \mathcal{I}_{v}^{*}(H) \rightarrow F_{1}$, such that $f_{*}(q)=f(q)^{e(q)}$ for all $q \in \mathfrak{X}(H)$. 
By construction of the $e(q)$ 's the following diagram is commutative

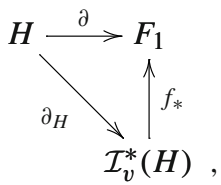

where $\partial_{H}$ is the canonical divisor theory of $H$, defined by $\partial_{H}(a)=a H$ for all $a \in H$. Therefore, $\partial$ is a divisor theory of $H$, and $f_{*}$ induces an isomorphism

$$
\mathcal{C}(\partial)=\operatorname{Coker}(\mathrm{q}(\partial)) \cong \operatorname{Coker}\left(\mathrm{q}\left(\partial_{H}\right)\right)=\mathcal{C}_{v}(H) .
$$

Let $G=\mathrm{q}(F) / \mathrm{q}(H)$ be the class group of $H \subset F$, and set $\bar{G}=\mathrm{q}\left(F_{0}\right) / \mathrm{q}(H) \subset G$. Obviously, we have

$$
\mathrm{q}(F) / \mathrm{q}\left(F_{0}\right) \cong \bigoplus_{q \in \mathfrak{X}(H)} \mathbb{Z} / e(q) \mathbb{Z} .
$$

Hence, we obtain an exact sequence

$$
0 \rightarrow \bar{G} \rightarrow G \rightarrow \bigoplus_{q \in \mathfrak{X}(H)} \mathbb{Z} / e(q) \mathbb{Z} \rightarrow 0 .
$$

Since $\partial=d_{\mid H}, \mathbf{q}(d)$ induces an epimorphism

$$
\delta: \bar{G}=\mathrm{q}\left(F_{0}\right) / \mathrm{q}(H) \rightarrow \operatorname{Coker}(\mathrm{q}(\partial)) \cong \mathcal{C}_{v}(H) .
$$

Thus we obtain the following result.

Proposition 3.6 Let all notation be as above.

1. $\mathcal{C}_{v}(H)$ is isomorphic to a subquotient of the class group $G$ of $H \hookrightarrow F$. In particular, we have

$$
r(G) \geq r\left(\mathcal{C}_{v}(H)\right) .
$$

2. If $r(G)=r\left(\mathcal{C}_{v}(H)\right)<\infty$, then $P_{0}=P, F_{1}=F_{0}$, and $\bar{G} \cong \mathcal{C}_{v}(H)$.

Proof 1 . This follows immediately from the above construction.

2. From the exact sequence (3.3) we get $\mathrm{r}(\bar{G})=\mathrm{r}(G)$. Hence $\mathrm{r}(\bar{G})=\mathrm{r}\left(\mathcal{C}_{v}(H)\right)$ by assumption.

We consider now the epimorphism $\delta: \bar{G} \rightarrow \mathcal{C}_{v}(H)$ from above. Since $\mathrm{r}\left(\mathcal{C}_{v}(H)\right)=\mathrm{r}(\bar{G})<$ $\infty, \delta \otimes \mathbb{Q}$ is an isomorphism, which implies that the kernel of $\delta$ consists only of elements with finite order.

Assume to the contrary that $P_{0} \neq P$, and let $p \in P \backslash P_{0}$. If $g$ is the image of $p$ in $\bar{G}$, we have by definition $\delta(g)=0$. Hence $g$ is of finite order, which means $p^{n} \in H$ for some $n \in \mathbb{N}$. From $H^{\times}=H \cap F^{\times}$, we obtain $p^{n} H \neq H$. Since $H \hookrightarrow F$ is a divisor homomorphism, we have $p^{n} H=p^{n} F \cap H$. Therefore $p^{n}$ is not irrelevant. From Lemma 3.5.1 we deduce that $p$ is not irrelevant. Since $p^{n} \in p F \cap H$, Lemma 3.5.2 tells us $q:=p F \cap H \in \mathrm{v}-\operatorname{spec}(H) \backslash\{\emptyset\}=\mathfrak{X}(H)$. From

$$
p^{n} \in q=f(q) F \cap H
$$

we obtain $p=f(q) \in P_{0}$, a contradiction. Now $P_{0}=P$ implies $F_{0}=F_{1}, \partial=H \hookrightarrow F_{0}$ and $\bar{G}=\operatorname{Coker}(q(\partial)) \cong \mathcal{C}_{v}(H)$. 
We apply this result now for block monoids. Let $G$ be an abelian group, $G_{0} \subset G$ a condensed subset. We assume that $\mathrm{r}\left(\mathcal{C}_{v}\left(\mathcal{B}\left(G_{0}\right)\right)\right)=\mathrm{r}\left(\left[G_{0}\right]\right)$. For $g \in G_{0}$ we set

$$
e_{G_{0}}(g)=\min v_{g}\left(\mathcal{B}\left(G_{0}\right)\right) \backslash\{0\}=\operatorname{gcd} v_{g}\left(\mathcal{B}\left(G_{0}\right)\right) .
$$

and define $G_{0}^{1}=\left\{e_{G_{0}}(g) g \mid g \in G_{0}\right\}$. Then in the notation from (3.6) we have $\mathcal{B}\left(G_{0}\right) \subset$ $F_{1}=\left[\left\{g^{e_{G_{0}}(g)}\right\}\right]$ and this inclusion $\iota$ is a divisor theory. Since $G_{0}$ is condensed, the sum homomorphism $\sigma: \mathcal{F}\left(G_{0}\right) \longrightarrow G$ induces an isomorphism $\mathcal{F}\left(G_{0}\right) / \mathcal{B}\left(G_{0}\right) \longrightarrow\left[G_{0}\right]$. By restriction we get an isomorphism $\bar{\sigma}: F_{1} / \mathcal{B}\left(G_{0}\right) \longrightarrow\left[G_{0}^{1}\right]$ with

$$
\bar{\sigma}\left(\left[\left\{g^{e_{G_{0}}(g)}\right] \mid g \in G_{0}\right\}\right)=G_{0}^{1} .
$$

By identifying the class group of $\iota$ with $\left[G_{0}^{1}\right]$ by means of $\bar{\sigma}$ we obtain the block homomorphism

$$
\beta_{G_{0}}: \mathcal{B}\left(G_{0}\right) \longrightarrow \mathcal{B}\left(G_{0}^{1}\right)
$$

defined by

$$
\prod_{g \in G_{0}} g^{a_{g}}=\prod_{g \in G_{0}}\left(g^{e_{G_{0}}(g)}\right)^{a_{g} / e_{G_{0}}(g)} \mapsto \prod_{g \in G_{0}}\left(e_{G_{0}}(g) g\right)^{a_{g} / e_{G_{0}}(g)} .
$$

Lemma 3.7 In the above situation the following are equivalent:

1. $\mathcal{B}\left(G_{0}\right) \hookrightarrow \mathcal{F}\left(G_{0}\right)$ is a divisor theory.

2. For all $g \in G_{0}$ we have $e_{G_{0}}(g)=1$.

3. $\left[G_{0}\right]=\left[G_{0}^{1}\right]$.

Proof $1 \Longrightarrow 2$ : Suppose that $\mathcal{B}\left(G_{0}\right) \hookrightarrow \mathcal{F}\left(G_{0}\right)$ is a divisor theory and let $g \in G_{0}$. Then $g=\operatorname{gcd}(X)$ for some $\emptyset \neq X \subset \mathcal{B}\left(G_{0}\right)$. In particular we have $v_{g}(B)=1$ for some $B \in \mathcal{B}\left(G_{0}\right)$. Hence $e_{G_{0}}(g)=1$.

$2 \Longrightarrow 3$ : By our identifications of the class groups of the inclusions $\mathcal{B}\left(G_{0}\right) \hookrightarrow F_{1}$, $\mathcal{B}\left(G_{0}\right) \hookrightarrow \mathcal{F}\left(G_{0}\right)$ the exact sequence (3.3) translates into the exact sequence

$$
0 \longrightarrow\left[G_{0}^{1}\right] \longrightarrow\left[G_{0}\right] \longrightarrow \bigoplus_{g \in G_{0}} \mathbb{Z} / e_{G_{0}}(g) \mathbb{Z} \longrightarrow 0 .
$$

$3 \Rightarrow 1$ : If $\left[G_{0}^{1}\right]=\left[G_{0}\right]$ we get by the identifications above $q\left(F_{1}\right) / q\left(\mathcal{B}\left(G_{0}\right)\right)=$ $q\left(\mathcal{F}\left(G_{0}\right)\right) / q\left(\mathcal{B}\left(G_{0}\right)\right)$. Therefore $q\left(F_{1}\right)=q\left(F_{0}\right)$. Looking at the the definition of $F_{1}$ one immediately deduces $\mathcal{F}\left(G_{0}\right)=F_{1}$. Since $\mathcal{B}\left(G_{0}\right) \hookrightarrow F_{1}$ is a divisor theory, we are ready.

Proof (Proof of Theorem 3.4) Let $H$ be reduced Krull monoid with finitely generated class group. Choose a divisor theory $H \hookrightarrow F=\mathcal{F}(P)$ with class group $G$. Let $\pi: F \rightarrow G$ be the canonical epimorphism, and set $G_{0}=\pi(P) \subset G$. By Lemma 3.2, $G_{0} \subset G$ is a condensed subset, $\mathcal{B}\left(G_{0}\right) \hookrightarrow \mathcal{F}\left(G_{0}\right)$ is a cofinal divisor homomorphism, and we consider the block homomorphism $\boldsymbol{\beta}_{H}: H \rightarrow \mathcal{B}(H):=\mathcal{B}\left(G_{0}\right)$. Since $\mathcal{B}\left(G_{0}\right)$ is again reduced and Krull, we can iterate this construction. Indeed, for $n \in \mathbb{N}_{0}$ we define inductively

$$
\begin{aligned}
\mathcal{B}^{0}(H)=H, \quad \mathcal{B}^{n+1}(H) & =\mathcal{B}\left(\mathcal{B}^{n}(H)\right), \quad \boldsymbol{\beta}_{H}^{0}=\operatorname{id}_{H}: H \rightarrow \mathcal{B}^{0}(H), \\
\boldsymbol{\beta}_{H}^{n+1} & =\boldsymbol{\beta}_{\mathcal{B}_{H}^{n}} \circ \boldsymbol{\beta}_{H}^{n}: H \rightarrow \mathcal{B}_{H}^{n+1} .
\end{aligned}
$$

For $n \in \mathbb{N}$, let $\bar{G}^{n}$ be the class group of $\mathcal{B}^{n-1}(H)$. Then, by construction, we have

$$
\mathcal{B}^{n}(H)=\mathcal{B}\left(\bar{G}_{0}^{n}\right)
$$


for a condensed subset $\bar{G}_{0}^{n} \subset \bar{G}^{n}$ such that $\left[\bar{G}_{0}^{n}\right]=\bar{G}^{n}$. It follows from Proposition 3.6.1 that we have a descending sequence of ranks:

$$
\mathrm{r}\left(\bar{G}^{1}\right) \geq \mathrm{r}\left(\bar{G}^{2}\right) \geq \mathrm{r}\left(\bar{G}^{3}\right) \geq \cdots .
$$

and that all $\bar{G}^{n}$ are finitely generated, too. The sequence of ranks stabilizes. We choose $k \in \mathbb{N}$ such $\mathrm{r}\left(\bar{G}^{n+1}\right)=\mathrm{r}\left(\bar{G}^{n}\right)$ for all $n \geq k$ and set $G^{n}=\bar{G}^{n+k}, G_{0}^{n}=\bar{G}_{0}^{k+n}$ for all $n \in \mathbb{N}_{0}$. Then we can assume that for all $n \in \mathbb{N}_{0}$ the block homomorphism $\mathcal{B}\left(G_{0}^{n}\right) \longrightarrow \mathcal{B}\left(G_{0}^{n+1}\right)$ is the block homomorphism $\beta_{G_{0}^{n}}$ constructed above. In particular we have

$$
G^{0}=\left[G_{0}^{0}\right] \supset G^{1}=\left[G_{0}^{1}\right] \supset \cdots \quad \text { and } G_{0}^{n+1}=\left\{e_{G_{0}^{n}}(g) g \mid g \in G_{0}^{n}\right\}
$$

for all $n \in \mathbb{N}_{0}$.

For all $n \in \mathbb{N}_{0}$ we define

$$
\gamma^{n, n+1}: G_{0}^{n} \longrightarrow G_{0}^{n+1}, \quad g \mapsto e_{G_{0}^{n}}(g) g, \quad \gamma^{n}=\gamma^{n, n-1} \circ \cdots \circ \gamma^{0,1}: G_{0}^{0} \longrightarrow G_{0}^{n}
$$

(in particular $\gamma^{0}=\operatorname{id}_{G_{0}^{0}}$ ). For $n \in \mathbb{N}_{0}$ and $g \in G_{0}^{0}$ we define inductively

$$
e^{0}(g)=1, \quad e^{n+1}(g)=e_{G_{0}^{n}}\left(\gamma^{n}(g)\right) e^{n}(g) .
$$

Then an easy induction shows for all $n \in \mathbb{N}_{0}$ :

- for all $g \in G_{0}$ we have $\gamma^{n}(g)=e^{n}(g) g$;

- the composition of block homomorphisms

$$
\beta^{n}: \mathcal{B}\left(G_{0}^{0}\right) \stackrel{\beta_{G_{0}^{0}}}{\longrightarrow} \mathcal{B}\left(G_{0}^{1}\right) \stackrel{\beta_{G_{0}^{0}}}{\longrightarrow} \ldots \stackrel{\beta_{G_{0}^{n-1}}}{\longrightarrow} \mathcal{B}\left(G_{0}^{n}\right)
$$

is given as follows: if $B=\prod_{g \in G_{0}} g^{a_{g}} \in \mathcal{B}\left(G_{0}\right)$ then

$$
\beta^{n}(B)=\prod_{h \in G_{0}^{n}} h^{b_{h}},
$$

where for each $h \in G_{0}^{n}$

$$
b_{h}=\sum_{\substack{g \in G_{0} \\ \gamma^{n}(g)=h}} \frac{a_{g}}{e^{n}(g)} .
$$

In particular

$$
\left|\beta^{n}(B)\right|=\sum_{g \in G_{0}^{0}} \frac{a_{g}}{e^{n}(g)} .
$$

Now let $B \in \mathcal{B}\left(G_{0}^{0}\right)$. Since for each $g \in G_{0}^{0}$ the sequence $\left(e^{n}(g)\right)_{n \in \mathbb{N}_{0}}$ is increasing, we get the decreasing sequence of positive integers

$$
|B| \geq\left|\beta^{1}(B)\right| \geq \cdots .
$$

This sequence has to stabilize. Using again, that $\left(e^{n}(g)\right)_{n \in \mathbb{N}_{0}}$ is increasing, we see that for each $g \in \operatorname{supp}(B)=\left\{g \in G_{0}^{0} \mid a_{g}>0\right\}$, that the sequence $\left(e^{n}(g)\right)_{n \in \mathbb{N}_{0}}$ is bounded. Since any $g \in G_{0}^{0}$ is contained in some $\operatorname{supp}(B), B \in \mathcal{B}\left(G_{0}\right)$, we see that $\left(e^{n}(g)\right)_{n \in \mathbb{N}_{0}}$ is bounded for any $g \in G_{0}^{0}$. We set $e^{\infty}(g)=\max \left\{e^{n}(g) \mid n \in \mathbb{N}_{0}\right\}$. Since $e^{0}(g) \mid e^{1}(g) \ldots$ we get $e^{n}(g) \mid e^{\infty}(g)$ for any $n \in \mathbb{N}$ and any $g \in G_{0}^{n}$. 
We define $G^{\infty}=\left\langle\left\{e_{G_{0}}^{\infty} g \mid g \in G_{0}\right\}\right\rangle$. Since $G^{0}=\left[G_{0}\right]$, the group $G^{0} / G^{\infty}$ is a finitely generated torsion group, hence finite.

We obtain in $G^{0}$ the descending sequence of subgroups

$$
G^{0} \supset G^{1} \supset G^{2} \supset \cdots \supset G^{\infty}
$$

Since $G^{0} / G^{\infty}$ is finite, we have $\left[G_{0}^{n}\right]=G^{n}=G^{n+1}=\left[G_{0}^{n+1}\right]$ for some $n \in \mathbb{N}$. Using 3.7 we see that $\mathcal{B}\left(G_{0}^{n}\right) \hookrightarrow \mathcal{F}\left(G_{0}^{n}\right)$ is a divisor theory.

It remains to verify the statements $1-3$.

1. This holds true by (3.1).

2. The asserted inequalities hold for block homomorphisms by [20, Theorem 3.4.10.2], whence they hold for compositions of block homomorphisms.

3. For a Krull monoid $S$ with block homomorphism $\boldsymbol{\beta}_{S}, S$ is locally tame if and only if $\boldsymbol{\beta}_{S}(S)$ is locally tame by [16, Proposition 3.3]. Since $\theta^{*}$ is a composition of block homomorphisms, it follows that $H$ is locally tame if and only if $\mathcal{B}\left(G_{0}^{*}\right)$ is locally tame.

Remark 3.8 (Uniqueness of transfer homomorphisms) Let $H$ be a Krull monoid. The divisor theory of $H$ is uniquely determined. Suppose that the class group of $H$ is torsion or finitely generated. Then Proposition 3.3 and Theorem 3.4 imply that there is a subset $G_{0}^{*}$ of an abelian group and a transfer homomorphism $\theta^{*}: H \rightarrow \mathcal{B}\left(G_{0}^{*}\right)$ such that the inclusion $\mathcal{B}\left(G_{0}^{*}\right) \hookrightarrow$ $\mathcal{F}\left(G_{0}^{*}\right)$ is a divisor theory. In contrast to the divisor theory of $H, G_{0}^{*}$ and $\theta^{*}$ are not uniquely determined in general. To give a simple example, consider a Krull monoid $H$ with infinite cyclic class group $G$, say $G=\langle e\rangle$, and suppose that $G_{P}=\{-2 e,-e, 0, e, 2 e\} \subset G$ is the set of classes containing prime divisors. By Lemma 3.2, $\boldsymbol{\beta}_{H}: H \rightarrow \mathcal{B}\left(G_{P}\right)$ is a transfer homomorphism, and by (2.2) the inclusion $\mathcal{B}\left(G_{P}\right) \hookrightarrow \mathcal{F}\left(G_{P}\right)$ is a divisor theory. By [4, Proposition 6.12], there is a transfer homomorphism $\theta: \mathcal{B}\left(G_{P}\right) \rightarrow \mathcal{B}\left(C_{3}\right)$, where $C_{3}$ is a cyclic group with three elements. Thus, $\theta^{*}=\theta \circ \boldsymbol{\beta}_{H}: H \rightarrow \mathcal{B}\left(C_{3}\right)$ is a transfer homomorphism and the inclusion $\mathcal{B}\left(C_{3}\right) \hookrightarrow \mathcal{F}\left(C_{3}\right)$ is a divisor theory.

This example shows in particular that the iteration process, given in (3.4), need not stabilize when the inclusion $\mathcal{B}\left(G_{0}^{n}\right) \hookrightarrow \mathcal{F}\left(G_{0}^{n}\right)$ is a divisor theory for the first time. The question, when this process stabilizes, was investigated in detail in [35], in case of torsion class groups.

Remark 3.9 (On global tameness) Let $H$ be a Krull monoid and let $\boldsymbol{\beta}_{H}: H \rightarrow \mathcal{B}\left(G_{P}\right)$ be the transfer homomorphism, given in Lemma 3.2. Then $H$ is locally tame if and only if $\mathcal{B}\left(G_{P}\right)$ is locally tame. If the class group is finitely generated, then $H$ is tame if and only if $G_{P}$ is finite; but this equivalence does not hold in general ([21, Theorem 4.2 and Example 4.13]).

Remark 3.10 (On the set of minimal distances) Let $H$ be an atomic monoid. The set of minimal distances $\Delta^{*}(H)$ is defined as the set of all min $\Delta(S)$ over all divisor-closed submonoid $S \subset H$ with $\Delta(S) \neq \emptyset$. It plays a crucial role in structural descriptions of sets of lengths (see [20, Chap. 4]). Suppose that $H$ is a Krull monoid with class group $G$ and suppose that each class contains a prime divisor. By [20, Proposition 4.3.13], we have

$$
\Delta^{*}(H)=\left\{\min \Delta\left(G_{0}\right) \mid G_{0} \subset G, \Delta\left(G_{0}\right) \neq \emptyset\right\} .
$$

Thus, when studying the set of minimal distances $\Delta^{*}(H)$ of a Krull monoid with class group $G$ and prime divisors in all classes, we need to study the set of distances $\Delta\left(G_{0}\right)$ of Krull monoids $\mathcal{B}\left(G_{0}\right)$, and for doing so we use Theorem 3.4 (see [20, Chap. 6.7], [25,32]). 


\section{Transfer Krull monoids and weakly Krull monoids}

Transfer Krull monoids are monoids that allow a (suitably defined) transfer homomorphism onto a monoid of zero-sum sequences. By Lemma 3.2, Krull monoids are transfer Krull, but transfer Krull monoids need neither be commutative, nor cancellative, nor completely integrally closed, nor $v$-noetherian. We start with the definition of a weak transfer homomorphism as given in [6, Definition 2.1].

Definition 4.1 Let $H$ be a monoid.

1. A monoid homomorphism $\theta: H \rightarrow B$ between atomic unit-cancellative monoids $H$ and $B$ is called a weak transfer homomorphism if it has the following two properties.

(T1) $B=B^{\times} \theta(H) B^{\times}$and $\theta^{-1}\left(B^{\times}\right)=H^{\times}$.

(WT2) If $a \in H, n \in \mathbb{N}, v_{1}, \ldots, v_{n} \in \mathcal{A}(B)$ and $\theta(a)=v_{1} \cdot \ldots \cdot v_{n}$, then there exist $u_{1}, \ldots, u_{n} \in \mathcal{A}(H)$ and a permutation $\tau \in \mathfrak{S}_{n}$ such that $a=u_{1} \cdot \ldots \cdot u_{n}$ and $\theta\left(u_{i}\right) \in B^{\times} v_{\tau(i)} B^{\times}$for each $i \in[1, n]$.

2. $H$ is said to be a transfer Krull monoid (over $G_{0}$ ) if $H$ is atomic and unit-cancellative and if there exists a weak transfer homomorphism $\theta: H \rightarrow \mathcal{B}\left(G_{0}\right)$ for a subset $G_{0}$ of an abelian group $G$. A domain is said to be transfer Krull if its monoid of regular elements is a transfer Krull monoid.

Let $\theta: H \rightarrow B$ be a homomorphism of atomic unit-cancellative monoids. If $\theta$ is a transfer homomorphism, then $\theta$ is a weak transfer homomorphism and the converse holds if $H$ and $B$ are both commutative ( $[6$, Sect. 2]). However, there are monoids which do not have a transfer homomorphism to any commutative monoid but which do have a weak transfer homomorphism to a Krull monoid ( [6, Remark 2.4] and [3, Example 4.5]).

\section{Example 4.2}

We mention some key examples of transfer Krull monoids that are not Krull, and refer to [26, Sect. 5] for more.

1. Let $R$ be a bounded hereditary noetherian prime ring. If all stably free right $R$-ideals are free, then $R$ is a transfer Krull domain ( [37, Theorem 4.4]). For more of this flavor see [6].

2. A Mori domain is Krull if and only if it completely integrally closed. There are results stating that Mori domains, that are close to their complete integral closure, are transfer Krull (for a sample, see [22, Theorem 5.8]).

3 . Let $R$ be a Bass ring and $\mathcal{C}$ be the class of finitely generated torsion-free $R$-modules. Then $\mathcal{V}(\mathcal{C})$ is a commutative, but not necessarily cancellative transfer Krull monoid over a subset of a finitely generated abelian group ( [5, Theorem 1.1]).

Theorem 4.3 Let $H$ be a transfer Krull monoid over a subset of an abelian group $G$ that is finitely generated resp. torsion. Then there exist an abelian group $G^{*}$ that is finitely generated resp. torsion, a subset $G_{0}^{*} \subset G^{*}$ such that $\mathcal{B}\left(G_{0}^{*}\right) \hookrightarrow \mathcal{F}\left(G_{0}^{*}\right)$ is a divisor theory, and a weak transfer homomorphism $\theta^{*}: H \rightarrow \mathcal{B}\left(G_{0}^{*}\right)$. In particular, we have

1. $\mathcal{L}(H)=\mathcal{L}\left(G_{0}^{*}\right)$.

2. If $H$ is commutative, then $H$ has finite catenary degree if and only if $\mathcal{B}\left(G_{0}^{*}\right)$ has finite catenary degree.

Remark 4.4 For cancellative but not necessarily commutative monoids $H$, axiomatically defined distance functions $d$ and associated catenary degrees $c_{d}(H)$ are studied in $[6$, Sect. 
4] and in [37]. The finiteness of these catenary degrees is also preserved under transfer homomorphisms, as it is the case in the commutative setting.

Proof Let $G$ be an abelian group that is finitely generated resp. torsion, $G_{0} \subset G$ be a subset, and $\theta_{1}: H \rightarrow \mathcal{B}\left(G_{0}\right)$ be a weak transfer homomorphism. By Theorem 3.4, resp. by Proposition 3.3, there exist a finitely generated abelian group $G^{*}$, resp. a torsion group $G^{*}$, a subset $G_{0}^{*} \subset G^{*}$ such that $\mathcal{B}\left(G_{0}^{*}\right) \hookrightarrow \mathcal{F}\left(G_{0}^{*}\right)$ is a divisor theory, and a transfer homomorphism $\theta_{2}: \mathcal{B}\left(G_{0}\right) \rightarrow \mathcal{B}\left(G_{0}^{*}\right)$. Then $\theta^{*}=\theta_{2} \circ \theta_{1}: H \rightarrow \mathcal{B}\left(G_{0}^{*}\right)$ is a weak transfer homomorphism. Thus, it remains to verify the in particular statements.

As in the case of transfer homomorphisms, $\mathcal{L}(H)=\mathcal{L}\left(G_{0}^{*}\right)$ follows easily from the definition. Now suppose that $H$ is commutative. Since $H$ is unit-cancellative, the proof runs along the same lines as in the cancellative setting ( [20, Theorem 3.2.5]).

Next we consider weakly Krull monoids and weakly Krull domains. Weakly Krull domains were introduced by Anderson et al. [1,2] and Halter-Koch showed their purely multiplicative character [28]. We refer to [29] for the ideal theory of weakly Krull monoids, to [22, Example 5.7] for an extended list of examples, and to [14] for weakly Krull monoid algebras. We recall the definition. A commutative and cancellative monoid is

- weakly Krull ([29, Corollary 22.5]) if

$$
H=\bigcap_{\mathfrak{p} \in \mathfrak{X}(H)} H_{\mathfrak{p}} \quad \text { and } \quad\{\mathfrak{p} \in \mathfrak{X}(H) \mid a \in \mathfrak{p}\} \text { is finite for all } a \in H .
$$

- weakly factorial if one of the following equivalent conditions is satisfied ([29, Exercise 22.5]):

- Every non-unit is a finite product of primary elements.

- $H$ is a weakly Krull monoid with trivial $t$-class group.

A commutative domain $D$ is weakly Krull resp. weakly factorial if its monoid of nonzero elements if weakly Krull resp. weakly factorial. The arithmetic of weakly Krull monoids is studied via $T$-block monoids ([20, Chap. 3.4]). To recall this concept, let $G$ be an additively abelian group, $G_{0} \subset G$ a subset, $T$ a commutative and cancellative monoid, $\sigma: \mathcal{F}\left(G_{0}\right) \rightarrow G$, the sum, and $\iota: G \rightarrow G$ a monoid homomorphism. Then

$$
B=\mathcal{B}\left(G_{0}, T, \iota\right)=\left\{S t \in \mathcal{F}\left(G_{0}\right) \times T \mid \sigma(S)+\iota(t)=0\right\} \subset \mathcal{F}\left(G_{0}\right) \times T=F
$$

is the $T$-block monoid over $G_{0}$ defined by $\iota$. The inclusion $B \hookrightarrow F$ is a divisor homomorphism, and if $G_{0}=G$ or if $G_{0} \cup \iota(T)$ consists of torsion elements, then $B \hookrightarrow F$ is cofinal.

Proposition 4.5 Let $D=\mathcal{F}(P) \times T$ be a reduced atomic monoid, where $P \subset D$ a set of primes and $T \subset D$ is a submonoid. Let $H \subset D$ be a submonoid such that $H \hookrightarrow D$ is a cofinal divisor homomorphism with class group $G=q(D) / q(H)$, and let $G_{P}=\{[p]=$ $p q(H) \mid p \in P\} \subset G$ be the set of classes containing primes. Let $\iota: T \rightarrow G$ be defined by $\iota(t)=[t], \quad F=\mathcal{F}\left(G_{P}\right) \times T, B=\mathcal{B}\left(G_{P}, T, \iota\right) \subset F$, and let $\widetilde{\boldsymbol{\beta}}: D \rightarrow F$ be the unique homomorphism satisfying $\widetilde{\boldsymbol{\beta}}(p)=[p]$ for all $p \in P$ and $\widetilde{\boldsymbol{\beta}} \mid T=i d_{T}$.

1. The restriction $\boldsymbol{\beta}=\widetilde{\boldsymbol{\beta}} \mid H: H \rightarrow B$ is a transfer homomorphism satisfying $c(H, \boldsymbol{\beta}) \leq 2$.

2. $B \hookrightarrow F$ is a cofinal divisor homomorphism, and there is an isomorphism $\bar{\psi}: q(F) / q(B) \rightarrow$ $G$, such that $\bar{\psi}(S t)=\sigma(S)+\iota(t)$ for all $S t \in \mathcal{F}\left(G_{P}\right) \times T$.

Proof 1. and 2. See [20, Proposition 3.4.8]. 
Theorem 4.6 Let $H$ be a v-noetherian weakly Krull monoid with $\emptyset \neq \mathfrak{f}=(H: \widehat{H}) \subsetneq H$ and suppose that $\mathcal{C}_{v}(H)$ is finite. Let

$$
\mathcal{P}^{*}=\{\mathfrak{p} \in \mathfrak{X}(H) \mid \mathfrak{p} \supset \mathfrak{f}\}, \mathcal{P}=\mathfrak{X}(H) \backslash \mathcal{P}^{*}, \text { and } T=\prod_{\mathfrak{p} \in \mathcal{P}^{*}}\left(H_{\mathfrak{p}}\right)_{\text {red }} .
$$

1. There is a transfer homomorphism $\beta: H \rightarrow \mathcal{B}\left(G_{\mathcal{P}}, T, \iota\right)$, where $G_{\mathcal{P}} \subset \mathcal{C}_{v}(H)$ is the set of classes containing minimal prime ideals. Moreover, $\mathcal{B}\left(G_{\mathcal{P}}, T, \iota\right)$ is a v-noetherian weakly Krull monoid.

2. For every divisor-closed submonoid $S \subset H$ with $v_{\mathfrak{p}}(a H)=0$ for all $a \in S$ and all $\mathfrak{p} \in \mathcal{P}^{*}$, there is a subset $G_{S}^{*}$ of $\mathcal{C}_{v}(H)$ such that $\mathcal{B}\left(G_{S}^{*}\right) \hookrightarrow \mathcal{F}\left(G_{S}^{*}\right)$ is a divisor theory and a transfer homomorphism $\theta_{S}^{*}: S \rightarrow \mathcal{B}\left(G_{S}^{*}\right)$.

Remark 4.7 Item 2 is of interest when studying the set $\Delta^{*}(H)$ of minimal distances of $H$ (see Remark 3.10 and [24]).

Proof 1. By (2.1), there is a cofinal divisor homomorphism $\mathcal{H} \rightarrow \mathcal{I}_{v}^{*}(H)$, where $\mathcal{H}=\{a H \mid$ $a \in H\}$ and $\mathcal{C}_{v}(H)=\mathrm{q}\left(\mathcal{I}_{v}^{*}(H)\right) / \mathrm{q}(\mathcal{H})$. By [22, Sect. 5], we have

$$
\mathcal{I}_{v}^{*}(H) \cong D=\mathcal{F}(\mathcal{P}) \times T .
$$

If $H_{0}$ denotes the image of $\mathcal{H}$ under this isomorphism, then $\mathcal{C}_{v}(H) \cong G:=\mathrm{q}(D) / \mathrm{q}\left(H_{0}\right)$. If $G_{\mathcal{P}}=\left\{[p]=p q\left(H_{0}\right) \mid p \in \mathcal{P}\right\} \subset G$ and $\iota: T \rightarrow G, \iota(t)=t \mathrm{q}\left(H_{0}\right)$, then Proposition 4.5.1 implies that $\boldsymbol{\beta}_{0}: H_{0} \rightarrow B=\mathcal{B}\left(G_{\mathcal{P}}, T, \iota\right)$ is a transfer homomorphism. The localizations $H_{\mathfrak{p}}$ are primary and $v$-noetherian for all $\mathfrak{p} \in \mathcal{P}^{*}$. Thus, $F=\mathcal{F}\left(G_{\mathcal{P}}\right) \times T$ is $v$-noetherian and $B$ is $v$-noetherian by [20, Proposition 2.4.4]. Since $\mathrm{q}(F) / \mathrm{q}(B) \cong G$ is finite, $B$ is weakly Krull by [22, Lemma 5.2].

2. Let $S \subset H$ be a divisor-closed submonoid with $\mathrm{v}_{\mathfrak{p}}(a H)=0$ for all $a \in S$ and all $\mathfrak{p} \in \mathcal{P}^{*}$. Then $\mathcal{S}=\{a H \mid a \in S\} \subset \mathcal{H}$ is a divisor-closed submonoid, and we denote by $S_{0} \subset H_{0}$ the image of $\mathcal{S}$ under the above isomorphism. There is a subset $P_{0} \subset P$ such that $S_{0} \hookrightarrow \mathcal{F}\left(P_{0}\right)$ is a cofinal divisor homomorphism, whence $S_{0}$ is a Krull monoid whose class group is a subgroup of $G$. Thus, the assertion follows from Proposition 3.3.

As mentioned in the Introduction, the refinement (done in Proposition 3.3 and in Theorem 3.4) of the block homomorphism (as given in Lemma 3.2) simplifies arithmetical investigations. Indeed, this was the original motivation for work in this direction, and it was used a lot for finite abelian groups (see the references given in the Introduction). We discuss here what is known in case of infinite groups. Let $G$ be an abelian group and $G_{0} \subset G$ be a subset. If $G_{0}$ is finite, then $\mathcal{B}\left(G_{0}\right)$ is finitely generated, which implies a variety of arithmetical finiteness results. In particular, $\mathcal{B}\left(G_{0}\right)$ is locally tame, has finite catenary degree, and sets of lengths are well-structured. On the other side of the spectrum, we know that if $G_{0}$ contains an infinite subgroup of $G$, then $\mathcal{B}\left(G_{0}\right)$ is not locally tame, its catenary degree is infinite, and every finite subset of $\mathbb{N}_{\geq 2}$ occurs as a set of lengths [31]. If $G_{0}$ is infinite but does not contain an infinite subgroup, then information on the arithmetic is available only in very special cases (e.g., $[18,21,27,30,38])$. The best understood case is when $G$ is an infinite cyclic group. In this case, there is a simple characterization of several arithmetical finiteness properties (including local tameness and the finiteness of the catenary degree) of $\mathcal{B}\left(G_{0}\right)$ in terms of $G_{0}[19$, Theorem 4.2].

We discuss two examples of subsets $G_{0} \subset \mathbb{Z}^{s}$ with $s \geq 2$. In Example 4.10 (where $s=2$ ), we use the strategy developed in Sect. 3 to obtain a transfer homomorphism to a monoid of zero-sum sequences over a subset of an infinite cyclic group. Thus, in that case Theorem 4.2 
of [19] yields information on the arithmetic. In the first example, it will turn out that $\mathcal{B}\left(G_{0}\right)$ is an inner direct product. If $\mathbf{0} \in G_{0}$, then the sequence $\mathbf{0} \in \mathcal{A}\left(G_{0}\right)$ is a prime element of $\mathcal{B}\left(G_{0}\right)$, whence $\mathcal{B}\left(G_{0}\right)=\mathcal{B}(\{\boldsymbol{0}\}) \mathcal{B}\left(G_{0} \backslash\{\mathbf{0}\}\right)$ is an inner direct product. Thus, when studying the structure of $\mathcal{B}\left(G_{0}\right)$, we may always assume that $\mathbf{0} \notin G_{0}$.

Example 4.8 Let $s \geq 2, k_{2}, \ldots, k_{s} \in \mathbb{Q} \backslash\{-1\}$, and $G_{0}=G_{1} \cup G_{2} \subset \mathbb{Z}^{s}$, where

$$
G_{1} \subset\left\{\boldsymbol{a}=\left(a_{1}, \ldots, a_{s}\right) \in \mathbb{Z}^{s} \mid a_{v}=k_{\nu} a_{1} \text { for all } v \in[2, s]\right\}
$$

and

$$
G_{2} \subset\left\{\boldsymbol{b}=\left(b_{1}, \ldots, b_{s}\right) \in \mathbb{Z}^{s} \backslash\{\boldsymbol{0}\} \mid b_{2}=-b_{1}\right\} .
$$

1. $G_{1} \cap G_{2}=\emptyset$ and $\mathcal{B}\left(G_{0}\right)=\mathcal{B}\left(G_{1}\right) \mathcal{B}\left(G_{2}\right)$ is an inner direct product.

2. $\mathcal{B}\left(G_{0}\right)$ is locally tame resp. has finite catenary degree if and only if $\mathcal{B}\left(G_{1}\right)$ and $\mathcal{B}\left(G_{2}\right)$ are locally tame resp. have finite catenary degree.

Remark 4.9 The same equivalence as stated in 2. holds true for several further arithmetical finiteness properties (see, for example, [18, Theorem 3.11]).

Proof 1. Let $S \in \mathcal{B}\left(G_{0}\right)$ be given. Then $S=S_{1} S_{2}$, where

$$
S_{1}=\prod_{\nu=1}^{l} \boldsymbol{a}_{\nu} \in \mathcal{F}\left(G_{1}\right) \text { and } S_{2}=\prod_{\nu=1}^{m} \boldsymbol{b}_{\nu} \in \mathcal{F}\left(G_{2}\right) .
$$

It suffices to show that $S_{1} \in \mathcal{B}\left(G_{1}\right)$. Since $S \in \mathcal{B}\left(G_{0}\right)$, we have

$$
\mathbf{0}=\sigma(S)=\sigma\left(S_{1}\right)+\sigma\left(S_{2}\right)=\boldsymbol{a}_{1}+\cdots+\boldsymbol{a}_{l}+\boldsymbol{b}_{1}+\cdots+\boldsymbol{b}_{m}
$$

and hence

$$
\begin{aligned}
& 0=a_{1,1}+\cdots+a_{l, 1}+b_{1,1}+\cdots+b_{m, 1} \text { and } \\
& 0=a_{1,2}+\cdots+a_{l, 2}+b_{1,2}+\cdots+b_{m, 2} .
\end{aligned}
$$

Adding the last two equations and using that $b_{j, 2}=-b_{j, 1}$ for all $j \in[1, m]$, we infer that $0=\left(a_{1,1}+\cdots+a_{l, 1}\right)+\left(a_{1,2}+\cdots+a_{l, 2}\right)=\left(a_{1,1}+\cdots+a_{l, 1}\right)+k_{2}\left(a_{1,1}+\cdots+a_{l, 1}\right)$, and hence $a_{1,1}+\cdots+a_{l, 1}=0$. Thus, for all $v \in[2, s]$, we infer that $a_{1, v}+\cdots+a_{l, v}=$ $k_{v}\left(a_{1,1}+\cdots+a_{l, 1}\right)=0$, and hence $\mathbf{0}=\boldsymbol{a}_{1}+\cdots+\boldsymbol{a}_{l}=\sigma\left(S_{1}\right)$.

2. This follows from 1. and from [20, Proposition 1.6.8].

Example 4.10 Let $G_{0}=G_{1} \cup\{\boldsymbol{a}\} \subset \mathbb{Z}^{2}$, where $G_{1} \subset \mathbb{N}_{0}^{2}$ and $\boldsymbol{a}=\left(a_{1}, a_{2}\right) \in \mathbb{Z}^{2}$, with $a_{1}<0$ and $a_{2}<0$, such that $\left[G_{0}\right]=\left\langle G_{0}\right\rangle$ (i.e., $G_{0}$ is condensed). Since $\left[G_{0}\right]=\left[G_{1}\right]+\mathbb{N}_{0} a$ and $\left\langle G_{0}\right\rangle=\left\langle G_{1}\right\rangle+\mathbb{Z} \boldsymbol{a}$ we obtain in particular

$$
\left[G_{1}\right]+\mathbb{Z} \boldsymbol{a}=\left\langle G_{1}\right\rangle+\mathbb{Z} \boldsymbol{a} .
$$

We write $F \in \mathcal{F}\left(G_{0}\right)$ in the form $F=a^{v_{a}(F)} F^{\prime}$ with $F^{\prime} \in \mathcal{F}\left(G_{1}\right)$. For every $B \in \mathcal{B}\left(G_{0}\right)$, we have

$$
\mathrm{v}_{\boldsymbol{a}}(B) \boldsymbol{a}=-\sigma\left(B^{\prime}\right) .
$$

Hence we get an injective homomorphism

$$
\varphi: \mathcal{B}\left(G_{0}\right) \rightarrow \mathcal{F}\left(G_{1}\right), \quad B \mapsto B^{\prime} .
$$


We set $H=\varphi\left(\mathcal{B}\left(G_{0}\right)\right)$, whence $\mathcal{B}\left(G_{0}\right) \cong H$, and $\Gamma=\left\langle G_{1}\right\rangle /\left(\left\langle G_{1}\right\rangle \cap \mathbb{Z} \boldsymbol{a}\right)$. We define $\bar{\sigma}: \mathcal{F}\left(G_{1}\right) \rightarrow \Gamma$ to be the composition of the sum homomorphism $\mathcal{F}\left(G_{1}\right) \rightarrow\left\langle G_{1}\right\rangle$ and the canonical homomorphism $\left\langle G_{1}\right\rangle \rightarrow \Gamma$. It follows from (4.1) that $\bar{\sigma}$ is onto.

We claim that $H=\bar{\sigma}^{-1}(0)$. The inclusion $\subset$ follows from (4.2). Conversely, let $F^{\prime} \in$ $\bar{\sigma}^{-1}(0)$. Then $\sigma\left(F^{\prime}\right)=k \boldsymbol{a}$ for some $k \in \mathbb{Z}$. Since $\boldsymbol{a} \in(-\mathbb{N})^{2}$ and $\sigma\left(F^{\prime}\right) \in \mathbb{N}_{0}^{2}$ we have $k \leq 0$. Then $a^{-k} F^{\prime} \in \mathcal{B}\left(G_{0}\right)$ and therefore $F^{\prime} \in H$.

To show that $H \hookrightarrow \mathcal{F}\left(G_{1}\right)$ is cofinal, let $F^{\prime} \in \mathcal{F}\left(G_{1}\right)$ be given. Choose $F^{\prime \prime} \in \mathcal{F}\left(G_{1}\right)$ such that $\bar{\sigma}\left(F^{\prime \prime}\right)=-\bar{\sigma}\left(F^{\prime}\right)$, whence $F^{\prime} F^{\prime \prime} \in H$.

From [20, Proposition 2.5.1] we obtain now: $H \hookrightarrow \mathcal{F}\left(G_{1}\right)$ is a cofinal divisor homomorphism and there is a monomorphism $s: \mathcal{F}\left(G_{1}\right) / H \rightarrow \Gamma$ such that

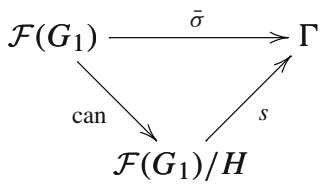

is commutative. Since $\bar{\sigma}$ is onto, $s$ is an isomorphism. Using the block homomorphism associated to the divisor homomorphism $H \hookrightarrow \mathcal{F}\left(G_{1}\right)$ and the isomorphisms $s$ and $\mathcal{B}\left(G_{0}\right) \cong$ $H$, we obtain a transfer homomorphism

$$
\mathcal{B}\left(G_{0}\right) \rightarrow \mathcal{B}\left(\bar{\sigma}\left(G_{1}\right)\right) .
$$

We consider two special cases. In both of them, it will turn out that the torsion-free rank of $\Gamma$ equals one.

Special Case 1. Let $G_{1}=\mathbb{N}_{0}^{2}$ and $\boldsymbol{a}=\left(a_{1}, a_{2}\right)$ with $a_{1}<0$ and $a_{2}<0$. Since $\mathbb{N}_{0}^{2}$ is a G-monoid (see [20, Chap. 2.7] for background on G-monoids) and $\mathbb{N}^{2}$ is the intersection of all non-empty prime $s$-ideals of $\mathbb{N}_{0}^{2}$, we have $\mathbb{N}_{0}^{2}+\mathbb{N}_{0} \boldsymbol{a}=\mathbb{Z}^{2}$. With all notation from above, we have that $\Gamma$ is a finitely generated group with torsion-free rank equal to 1 . Since $\sigma\left(\mathcal{F}\left(\mathbb{N}_{0}^{2}\right)\right)=\mathbb{N}_{0}^{2}$ and $\mathbb{Z}^{2}=\left\langle\mathbb{N}_{0}^{2}\right\rangle$, we obtain in this case a transfer homomorphism

$$
\mathcal{B}\left(G_{0}\right) \rightarrow \mathcal{B}(\Gamma)
$$

Special Case 2. Let $G_{1}$ be set of all $(m, n) \in \mathbb{N}_{0}^{2}$ such that $n \geq m^{2}$. We set $\boldsymbol{a}=(-1,-2)$. From

$$
\{(0,1),(1,1)\} \subset G_{1} \subset\left\{(m, n) \in \mathbb{N}_{0}^{2} \mid n \geq m\right\}=[(0,1),(1,1)]
$$

we obtain

$$
\left[G_{1}\right]=[(0,1),(1,1)] .
$$

Note that $[(0,1),(1,1)]$ is a factorial G-monoid. Arguing as in example before we obtain $\left[G_{1}\right]+\mathbb{N}_{0} \boldsymbol{a}=\mathbb{Z}^{2}$, which in particular implies that, $G_{0}$ is condensed. The homomorphism

$$
\left\langle G_{1}\right\rangle=\mathbb{Z}^{2} \rightarrow \mathbb{Z},(u, v) \mapsto 2 u-v
$$

has kernel $\mathbb{Z} \boldsymbol{a}$. Hence we obtain an isomorphism $\varphi: \Gamma \rightarrow \mathbb{Z}$ such that $\varphi\left(\bar{\sigma}\left(G_{1}\right)\right)=\varphi\left(G_{1}\right)$. We show $\varphi\left(G_{1}\right)=-\mathbb{N}_{0} \cup\{1\}$. For all $n \in \mathbb{N}_{0}$, we have $(0, n) \in G_{1}$ and hence $-n=$ $\varphi(0, n) \in \varphi\left(G_{1}\right)$. It remains to show that $\varphi\left(G_{1}\right) \cap \mathbb{N}=\{1\}$. Let $(n, m) \in G_{1}$ be such that $\varphi(m, n)=2 m-n>0$. Then $m^{2} \leq n<2 m$ which implies $m<2$. If $m=0$, then $n<0$, contradiction. If $m=1$ then $n=1$ and $\varphi(m, n)=1$. 
Acknowledgements This work was supported by the Austrian Science Fund FWF, Project P33499-N.

Funding Open access funding provided by Austrian Science Fund (FWF).

\section{Declarations}

Conflict of interest On behalf of all authors, the corresponding author states that there is no conflict of interest.

Open Access This article is licensed under a Creative Commons Attribution 4.0 International License, which permits use, sharing, adaptation, distribution and reproduction in any medium or format, as long as you give appropriate credit to the original author(s) and the source, provide a link to the Creative Commons licence, and indicate if changes were made. The images or other third party material in this article are included in the article's Creative Commons licence, unless indicated otherwise in a credit line to the material. If material is not included in the article's Creative Commons licence and your intended use is not permitted by statutory regulation or exceeds the permitted use, you will need to obtain permission directly from the copyright holder. To view a copy of this licence, visit http://creativecommons.org/licenses/by/4.0/.

\section{References}

1. Anderson, D.D., Anderson, D.F., Zafrullah, M.: Atomic domains in which almost all atoms are prime. Commun. Algebra 20, 1447-1462 (1992)

2. Anderson, D.D., Mott, J., Zafrullah, M.: Finite character representations for integral domains. Boll. Union Mat. Ital. 6, 613-630 (1992)

3. Bachman, D., Baeth, N., Gossell, J.: Factorizations of Upper Triangular Matrices. Linear Algebra Appl. 450, 138-157 (2014)

4. Baeth, N.R., Geroldinger, A.: Monoids of modules and arithmetic of direct-sum decompositions. Pacific J. Math. 271, 257-319 (2014)

5. Baeth, N.R., Smertnig,D.: Lattices over Bass rings and graph agglomerations. Algebras Represent. Theory (to appear). https://doi.org/10.1007/s10468-021-10040-2

6. Baeth, N.R., Smertnig, D.: Factorization theory: from commutative to noncommutative settings. J. Algebra 441, 475-551 (2015)

7. Baginski, P., Chapman, S.T., Rodriguez, R., Schaeffer, G.J., She, Y.: On the delta set and catenary degree of Krull monoids with infinite cyclic divisor class group. J. Pure Appl. Algebra 214, 1334-1339 (2010)

8. Baginski, P., Kravitz, R.: A new characterization of half-factorial Krull monoids. J. Algebra Appl. 9, 825-837 (2011)

9. Caminata, A.: Realization of Semigroups of Modules in Dimension Greater than Two. arXiv: 1910.11586

10. Facchini, A.: Direct sum decomposition of modules, semilocal endomorphism rings, and Krull monoids. J. Algebra 256, 280-307 (2002)

11. Facchini, A.: Krull monoids and their application in module theory. In: Facchini, A., Fuller, K., Ringel, C.M., Santa-Clara, C. (eds.) Algebras, Rings and their Representations, pp. 53-71. World Scientific, New Jersey (2006)

12. Facchini, A.: Direct-sum decompositions of modules with semilocal endomorphism rings. Bull. Math. Sci. 2, 225-279 (2012)

13. Facchini, A.: Semilocal Categories and Modules with Semilocal Endomorphism Rings. Progress in Mathematics, vol. 331. Birkhäuser/Springer, Cham (2019)

14. Fadinger, V., Windisch, D.: A Characterization of Weakly Krull Monoid Algebras. arXiv: 2009.04182

15. Fan, Y., Tringali, S.: Power monoids: a bridge between factorization theory and arithmetic combinatorics. J. Algebra 512, 252-294 (2018)

16. Gao, W., Geroldinger, A., Schmid, W.A.: Local and global tameness in Krull monoids. Commun. Algebra 43, 262-296 (2015)

17. García-Elsener, A., Lampe, P., Smertnig, D.: Factoriality and class groups of cluster algebras. Adv. Math. 358, 106858 (2019)

18. Geroldinger, A., Grynkiewicz, D.J.: On the arithmetic of Krull monoids with finite Davenport constant. J. Algebra 321, 1256-1284 (2009)

19. Geroldinger, A., Grynkiewicz, D.J., Schaeffer, G.J., Schmid, W.A.: On the arithmetic of Krull monoids with infinite cyclic class group. J. Pure Appl. Algebra 214, 2219-2250 (2010)

20. Geroldinger, A., Halter-Koch, F.: Non-Unique Factorizations. Algebraic, Combinatorial and Analytic Theory. Pure and Applied Mathematics, vol. 278. Chapman \& Hall/CRC, Boca Raton (2006) 
21. Geroldinger, A., Kainrath, F.: On the arithmetic of tame monoids with applications to Krull monoids and Mori domains. J. Pure Appl. Algebra 214, 2199-2218 (2010)

22. Geroldinger, A., Kainrath, F., Reinhart, A.: Arithmetic of seminormal weakly Krull monoids and domains. J. Algebra 444, 201-245 (2015)

23. Geroldinger, A., Ruzsa, I.: Combinatorial Number Theory and Additive Group Theory. Advanced Courses in Mathematics. CRM Barcelona, Birkhäuser (2009)

24. Geroldinger, A., Zhong, Q.: The set of distances in seminormal weakly Krull monoids. J. Pure Appl. Algebra 220, 3713-3732 (2016)

25. Geroldinger, A., Zhong, Q.: The set of minimal distances in Krull monoids. Acta Arith. 173, 97-120 (2016)

26. Geroldinger, A., Zhong, Q.: Factorization theory in commutative monoids. Semigroup Forum 100, 22-51 (2020)

27. Grynkiewicz, D.J.: The Characterization of Finite Elasticities. arXiv: 2012.12757

28. Halter-Koch, F.: Divisor theories with primary elements and weakly Krull domains. Boll. Un. Mat. Ital. B 9, 417-441 (1995)

29. Halter-Koch, F.: Ideal Systems. An Introduction to Multiplicative Ideal Theory. Marcel Dekker, New York (1998)

30. Hassler, W.: Factorization properties of Krull monoids with infinite class group. Colloq. Math. 92, 229242 (2002)

31. Kainrath, F.: Factorization in Krull monoids with infinite class group. Colloq. Math. 80, 23-30 (1999)

32. Plagne, A., Schmid, W.A.: On congruence half-factorial Krull monoids with cyclic class group. J. Combinat. Algebra 3, 331-400 (2020)

33. Schmid, W.A.: Arithmetic of block monoids. Math. Slov. 54, 503-526 (2004)

34. Schmid, W.A.: Differences in sets of lengths of Krull monoids with finite class group. J. Théor. Nombr. Bordx. 17, 323-345 (2005)

35. Schmid, W.A.: Higher-order class groups and block monoids of Krull monoids with torsion class group. J. Algebra Appl. 9, 433-464 (2010)

36. Schmid, W.A.: Some recent results and open problems on sets of lengths of Krull monoids with finite class group. In: Multiplicative Ideal Theory and Factorization Theory, pp. 323-352. Springer (2016)

37. Smertnig, D.: Factorizations in bounded hereditary noetherian prime rings. Proc. Edinburgh Math. Soc. 62, 395-442 (2019)

38. Zeng, X., Deng, G.: Elasticities of Krull monoids with infinite cyclic class group. J. Commutative Algebra 12,(2020)

Publisher's Note Springer Nature remains neutral with regard to jurisdictional claims in published maps and institutional affiliations. 